\title{
Caractérisation du profil aromatique de différentes variétés d'ananas par SPME en espace de tête / GC-FID
}

\author{
Alain SOLER $^{a *}$, Marc LebRUN ${ }^{b}$, Marie-Pierre BEAUTÉ ${ }^{a}$
}

\author{
a Cirad, UPR Système bananes \\ et ananas, PRAM, BP 214, \\ Fort-de-France, Martinique, \\ F-97285 France \\ alain.soler@cirad.fr \\ b Cirad, Département Flhor, \\ UPR qualité des aliments, \\ TA 50 / PS4, \\ Blvd. de La Lironde, \\ Montpellier Cedex, \\ F-34398 France
}

* Correspondance et tirés à part

Reçu le 27 Septembre 2004 Accepté le 26 octobre 2005

Fruits, 2006, vol. 61, p. 185-195 (c) 2006 Cirad/EDP Sciences All rights reserved DOI: 10.1051/fruits:2006016 www.edpsciences.org

RESUMEN Español, p. 195

\section{Characterization of the aromatic profile of different pineapple varieties assessed by SPME in GC-FID headspace.}

Abstract - Introduction. Among pineapple varieties (Ananas comosus [L.] Merr.), variability
in sensory characteristics is low between clones but it is marked between varieties. Ten Cayenne
$\times$ Perolera hybrids were selected by Cirad for commercial purposes. They presented aromatic
profiles distinct from the parents. The pineapple must be collected at the green-ripe stage to
be able to arrive in good condition on the consuming markets. Since the flavor released can
influence the consumer to buy the fresh fruit, we studied, in different varieties and hybrids, the
impact of the maturation stage of the fruit harvested and of the storage conditions on the volatile
compound emission. Materials and methods. The differences in volatile compound content
between the pineapple varieties were measured by extraction in headspace by SPME (PDMS
$100 \mu$ m fiber), followed by CPG/FID analysis on a Stabilwax column. The analyses were related
to the fruits of four or five clones of the varieties Cayenne, Perolera, Queen and Rondon, as well
as five selected hybrids by Cirad and another commercial hybrid, MD2. The impact of the matu-
rity stage (soluble solids and acidity) and of the fruit storage conditions on the volatile com-
pounds was assessed. Results and discussion. The aromatic profiles obtained for the clones
of the same variety showed a certain similarity and the differences between varieties appeared
repeatable. During maturation, an increase in the volatile compound emission occurred before
full maturation. Storage in cold conditions between $13{ }^{\circ} \mathrm{C}$ and $24{ }^{\circ} \mathrm{C}$ caused a significant increase
in the aromatic emission. Conclusion. To differentiate the aromatic profiles by SPME /GC-FID
from different varieties of pineapple seems possible in spite of their great similarity. Analysis
by GC-MS would allow the better characterization of the volatile pineapple compounds of fruits
of various origins.

Martinique / Ananas comosus / variety trials / gas chromatography / volatile compounds / maturation / cold storage

Caractérisation du profil aromatique de différentes variétés d'ananas par SPME en espace de tête / GC-FID.

Résumé - Introduction. Chez Ananas comosus, les variations entre clones des caractéristiques organoleptiques sont faibles ; celles entre variétés sont plus marquées. Une dizaine d'hybrides Cayenne $\times$ Perolera a été sélectionnée par le Cirad pour être développée commercialement. Ils présentent des profils aromatiques distincts des parents. L'ananas doit être récolté relativement vert pour pouvoir parvenir en bon état sur les marchés consommateurs. L'arôme dégagé pouvant inciter le consommateur à acheter le fruit frais, nous avons étudié, sur différentes variétés et hybrides, l'impact du stade de maturation du fruit à la récolte et de la durée de conservation sur l'émission des composés volatils. Matériel et méthodes. Les différences de teneurs en composés volatils entre les variétés d'ananas ont pu être mesurées par extraction en espace de tête par SPME (fibre PDMS $100 \mu \mathrm{m}$ ), suivie d'une analyse CPG/FID sur colonne Stabilwax. Les analyses ont porté sur les fruits de quatre ou cinq clones de chacune des variétés Cayenne, Perolera, Queen, Rondon, ainsi que de cinq hybrides Cirad et d'un hybride MD2. L'impact du stade de maturité (extrait sec et acidité libre) et des conditions de conservation sur les composés volatils a été évalué. Résultats et discussion. Les profils aromatiques obtenus pour les clones d'une même variété ont été très semblables et les différences intervariétales se sont révélées répétables. Au cours de la maturation, une augmentation de l'émission des composés volatils s'est produite avant la pleine maturation. La conservation au froid entre $13{ }^{\circ} \mathrm{C}$ et $24{ }^{\circ} \mathrm{C}$ a provoqué une forte augmentation de l'émission aromatique. Conclusion. Différencier les profils aromatiques par SPME/GC-FID de différentes variétés d'ananas semble possible malgré leur grande similitude. Une identification plus poussée, par GC-MS, des composés volatils d'ananas de différentes origines pourrait permettre de mieux préciser leur caractérisation.

Martinique / Ananas comosus / essai de variété / chromatographie en phase gazeuse / composé volatil / maturation / stockage au froid 


\section{Introduction}

Le Cirad a réuni en Martinique une collection de 585 accessions du genre Ananas. De nombreuses sélections des principales variétés comestibles (Ananas comosus) y figurent dont Queen, Rondon, Perolera, Cayenne lisse, ainsi que des hybrides issus de croisements entre Cayenne et Perolera [1, 2]. Ces différentes variétés se différentient par leurs caractères morphologiques, leur comportement agronomique, l'aspect de leurs fruits (forme, couleur de la peau et de la chair) et leurs qualités organoleptiques habituellement mesurées par des indicateurs simples liés à la maturation : extraits secs solubles (ESS), teneurs en acidité libre et acide ascorbique.

L'ananas n'est pas un fruit très aromatique puisqu'il présente environ dix fois moins d'émission de composés volatils que la banane et deux fois moins que le melon [3]. Parmi les composés importants déjà identifiés [4, 5], des esters aliphatiques et des hydroxy et acétoxy esters représentent près de la moitié de la concentration en composés volatils mesurée dans les fruits mûrs. Ces composés sont retrouvés chez l'hybride Flhoran 41 [6] sélectionné par le Cirad. Des terpènes comme le limonène, dont la concentration diminue avec la maturation, sont également présents chez l'ananas. Les thioesters, méthyl 3-(méthylthio) propanoate et éthyl 3-(méthylthio) propanoate contribueraient à l'odeur caractéristique de son fruit [7], ainsi que des composés dérivés furaniques comme le 2,5-diméthyl-4-hydroxy-3[2H]furanone [8]. Ainsi, les différences entre

Tableau I.

Répartition des clones considérés au sein de variétés d'Ananas comosus analysées quant à la production de composés volatils par leurs fruits au cours de leur maturation (Martinique).

$\begin{array}{lc}\text { Variété } & \text { Clone } \\ \text { Cayenne lisse } & \text { Ca1, Ca2, Ca3, Ca4, Ca5 } \\ \text { Perolera } & \text { Pe1, Pe2, Pe3, Pe4 } \\ \text { Queen } & \text { Qu1, Qu2, Qu3, Qu4, Qu5 } \\ \text { Rondon } & \text { Ro1, Ro2, Ro3, Ro4, Ro5 } \\ \text { Hybrides Cayenne x Perolera } & \text { Hy1, Hy2, Hy3, Hy4, Flhoran } 41 \\ \text { Hybride autre origine } & \text { MD2 }\end{array}$

variétés pourraient être suffisamment marquées pour justifier une utilisation comme critère de différentiation dans l'établissement des certificats d'obtention végétale (COV), critères qui sont essentiellement basés pour l'instant sur des caractéristiques botaniques classiques [9].

L'ananas, fruit non climactérique, doit être récolté relativement vert pour pouvoir être transporté, par voie maritime, des zones de production tropicales vers les marchés consommateurs. Dans ce contexte, il est apparu nécessaire de pouvoir évaluer l'impact du stade de maturation à la récolte, des systèmes de conservation par le froid ou de la durée de conservation [10] sur l'émission des composés volatils par le fruit, puisqu'en dehors de l'aspect visuel seul l'arôme dégagé peut inciter le consommateur à acheter le fruit frais.

L'extraction des arômes de fruits se fait de façon classique avec des solvants liquides (mélanges azéotropes), mais récemment une méthode dite SPME (Solid Phase Micro Extraction) a été développée. Elle permet de piéger les composés volatils dans l'espace de tête sans prétraitement des échantillons de fruits [11-15].

\section{Matériel et méthodes}

\subsection{Matériel végétal}

Les fruits de quatre variétés d'A. comosus (Cayenne lisse, Perolera, Queen et Rondon), les fruits de cinq hybrides, dont le Flhoran 41, sélectionnés par le Cirad-Flhor au sein d'une descendance Cayenne $\times$ Perolera et enfin un hybride d'une autre origine, le MD2, qui a pris une place prépondérante sur les marchés ces dernières années, ont été retenus pour les analyses aromatiques. Pour chacune des variétés d'A. comosus, de quatre à cinq clones ont été utilisés (tableau I).

Les ananas étudiés ont été cultivés sur la station expérimentale du Cirad à RivièreLézarde en Martinique (climat tropical, $15^{\circ}$ lat. $\mathrm{N}, 62^{\circ}$ long. O) sur des sols à halloysite. Les pratiques culturales classiques 
incluent un amendement de fond en $\mathrm{Ca}$ et $\mathrm{Mg}$, une fertilisation à base d'urée et de sulfate de potasse $\left[\left(\mathrm{K}_{2} \mathrm{O} / \mathrm{N}\right)=1,8\right]$ et un complément en fer sous forme de chélate. Les fruits ont été récoltés entre (135 et 158) jours après induction florale des plants en fonction de la vitesse de maturation des variétés. Le stade de maturité a été déterminé par la coloration externe des fruits, puis précisé par leur taux d'extraits secs solubles (ESS en ${ }^{\circ}$ Brix) et par la mesure de l'acidité des jus. Chaque échantillon a été constitué à partir du mélange de pulpes de trois à cinq fruits selon les clones étudiés.

\subsection{Préparation des échantillons}

Seule la partie médiane du fruit épluché a été utilisée pour les analyses. Pour les mesures d'extraits secs solubles (ESS en ${ }^{\circ}$ Brix), d'acidité libre et d'acide ascorbique, le jus a été extrait par pressage, sur étamine, de deux secteurs opposés de la pulpe. Pour l'analyse des composés volatils, $5 \mathrm{~g}$ de pulpe provenant de trois zones (près de la peau, au milieu de la chair et près du couur) ont été découpés, sur un autre secteur du fruit, en petits cubes de $5 \mathrm{~mm}$ de côté.

\subsection{Extraction des composés volatils par SPME}

L'extraction par SPME des composés volatils dans l'espace de tête (flacons de $20 \mathrm{~mL}$ ) a été faite avec des fibres PDMS (Supelco) pour passeur d'échantillon automatique CombiPal. Les échantillons ont été préchauffés $5 \mathrm{~min}$ à $45^{\circ} \mathrm{C}$. L'extraction des composés volatils en espace de tête a duré $30 \mathrm{~min}$. Les conditions d'extraction ont fait l'objet d'une mise au point préalable. La désorption dans l'injecteur a duré $30 \mathrm{~s}$ à $250^{\circ} \mathrm{C}$, avec un liner droit - diamètre interne de $0,75 \mathrm{~mm}$. La fibre a été nettoyée par désorption pendant $10 \mathrm{~min}$ à $220^{\circ} \mathrm{C}$ sous courant d'azote (pureté $99.9 \%$ ) avant chaque extraction.

\subsection{Analyse CPG / FID}

L'analyse par chromatographie en phase gazeuse (CPG) avec un détecteur à ionisa- tion de flamme (FID) a été faite sur colonne Stabilwax $30 \mathrm{~m}$, de diamètre interne $0,25 \mathrm{~mm}$, une épaisseur de phase de 0,25 $\mu \mathrm{m}$ et avec une pré-colonne (Restek) ; détection FID à $245^{\circ} \mathrm{C}$; injection à $250^{\circ} \mathrm{C}$ split fermé. Le temps utile d'analyse a été de $25 \mathrm{~min}$, la température initiale de $50{ }^{\circ} \mathrm{C}$, la programmation de $6{ }^{\circ} \mathrm{C}$ par min, la température finale de $220^{\circ} \mathrm{C}$, maintenue $5 \mathrm{~min}$ en isotherme ; gaz vecteur $\mathrm{He}=1,0 \mathrm{~mL} \cdot \mathrm{min}^{-1}$; air $=300 \mathrm{~mL} \cdot \mathrm{min}^{-1} ; \mathrm{H}_{2}=30 \mathrm{~mL} \cdot \mathrm{min}^{-1}$.

\subsection{Analyse CPG / MS}

L'analyse par chromatographie en phase gazeuse (CPG) avec spectrographie de masse (MS) a été effectuée dans les mêmes conditions que celle de la CPG / FID.

\subsection{Analyses des indicateurs de maturité}

Les solides solubles (ESS en ${ }^{\circ}$ Brix) ont été déterminés à l'aide d'un réfractomètre à compensation de température. L'acidité libre a été mesurée par titration à la soude $0,1 \mathrm{~N}$ sur $10 \mathrm{~mL}$ de jus et exprimée en $\mathrm{mEq} \cdot 100 \mathrm{~mL}^{-1}$. La teneur en acide ascorbique a été mesurée par la méthode au dichlorophénol indophénol.

\subsection{Traitements appliqués aux ananas}

L'évolution de l'émission des composés volatils au cours de la maturation naturelle des fruits sur pied ( $147 \mathrm{j}, 151 \mathrm{j}, 155 \mathrm{j}$ et $158 \mathrm{j}$ après le traitement d'induction florale pour l'hybride Flhoran 41) ou lors de leur conservation au froid a été suivie sur plusieurs clones par variété, mais les observations rapportées ne concernent qu'un seul clone par variété en raison de la similitude des évolutions observées au sein d'une même variété. Les vitesses de floraison et de maturation sur pied sont très différentes suivant les variétés : il y a plus de 3 semaines d'écart entre les plants de Queen précoces et ceux de Cayenne tardifs; le choix des stades de maturité à comparer a donc reposé sur les indicateurs de maturation. 


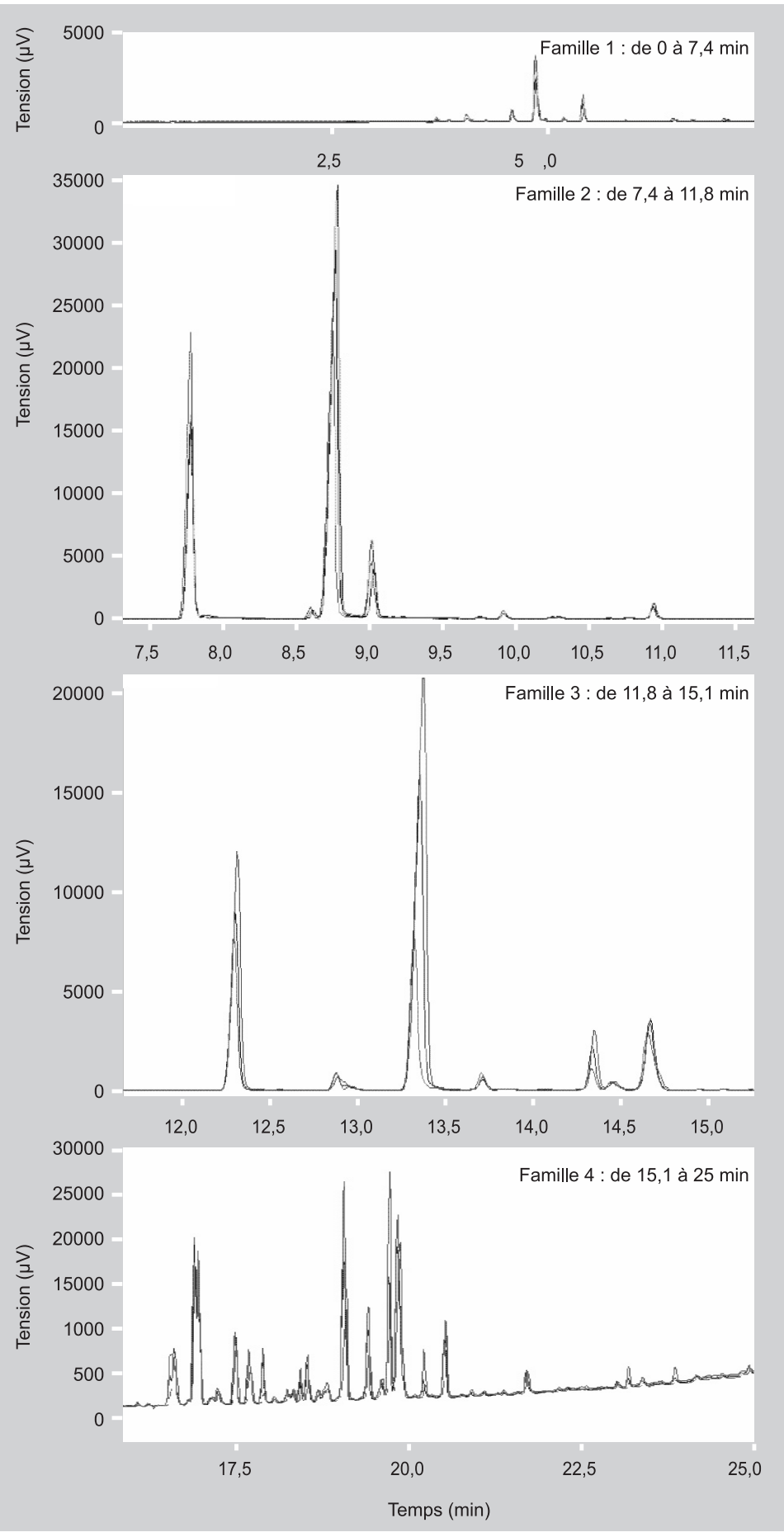

Figure 1.

Comparaison des profils aromatiques de trois clones (Qu2, Qu4 et Qu5) de la variété Queen (Ananas comosus), pour quatre familles de composés volatils définies par découpage du chromatogramme obtenu après analyse des fruits par SPME en fonction du temps de rétention. La superposition des trois profils révèle l'homogénéité intravariété du Queen.
Les ananas ont été conservés au froid pendant $25 \mathrm{j}$, selon des séquences de températures précises, ces conditions simulant les différentes étapes logistiques de commercialisation de fruits exportés par bateau (conteneurs, transport terrestre, grande distribution). Les observations ont été faites sur les fruits à la récolte (témoin), après $13 \mathrm{j}$ à $18{ }^{\circ} \mathrm{C}$, après $7 \mathrm{j}$ supplémentaires à $13^{\circ} \mathrm{C}$ et, enfin, après 5 autres jours à $24^{\circ} \mathrm{C}$.

Par ailleurs, l'impact d'un effet " terroir" a été évalué sur l'hybride Flhoran 41 cultivé dans deux localités différentes caractérisées par deux altitudes de culture et deux sols différents (altitude de $60 \mathrm{~m}$ sur sol à halloysite et de $125 \mathrm{~m}$ sur sol de ponce).

\section{Résultats et discussion}

L'objectif de l'étude ayant été de comparer des profils aromatiques des différentes variétés d'ananas et de leurs clones, l'analyse des chromatogrammes a été faite en les découpant en quatre familles de composés volatils en fonction de leur temps de rétention et donc de la volatilité et/ou la taille des molécules : famille F1 caractérisée par des temps de rétention de (0 à 7,4) min ; F2, de $(7,4$ à 11,8) $\min ; \mathrm{F} 3$, de (11,8 à 15,1) min ; $\mathrm{F} 4$, de (15,1 à 25) min (figure 1). Une identification partielle par chromatographie gazeuse avec spectrographie de masse (GC / MS) a été réalisée préalablement pour déterminer la nature des principaux composés aromatiques de chaque famille (tableau II).

L'extraction par un mélange azéotrope pentane/éther a permis d'extraire de la pulpe plus de 150 composés plus ou moins volatils qui participent à l'arôme de l'ananas [16]. L'extraction en espace de tête par SPME n'a certainement pas été aussi exhaustive, mais elle a permis d'extraire les composés les plus volatils de l'arôme d'ananas de manière simple et reproductible.

Les profils aromatiques des différentes variétés d'ananas obtenus par SPME et GC ont été similaires mais facilement différentiables. À maturité égale, les variations ont été liées à la présence ou non et à l'intensité d'un assez petit nombre de composés sur la centaine de pics significatifs. 


\subsection{Variations inter- et intravariétales pour chaque famille de composés volatils}

La famille F1, caractérisée par des temps de rétention de $(0$ à 7,4$)$ min, n'a représenté que $4 \%$ de la surface totale d'intégration (SI) pour les composés volatils identifiés dans les fruits de la variété Cayenne lisse et 6,6 \% SI pour les fruits de Rondon, avec une bonne homogénéité intravariétale (tableau III). Chez les fruits de Queen la famille F1 représente (4 à 5) \% SI, sauf pour ceux du clone Qu1 (10\% SI). Pour les ananas Perolera, les valeurs ont varié de (4 à 25) \% SI, révélant une hétérogénéité intravariétale plus forte (tableau III). Pour le clone Pe4 de cette même variété, le pic $\mathrm{n}^{\circ} 8$, au temps de rétention de 4,8 min représente $12,59 \%$ SI. Il correspond à une émission de butyrate d'éthyle nettement plus importante que pour les autres variétés (tableau IV). Pour les hybrides, la variabilité est relativement forte, de (1 à 12) \% SI, mais rappelons que ce ne sont pas des clones d'une même variété mais des individus issus du même croisement Cayenne $\times$ Perolera (tableau III). La majorité des pics formant la famille F1 ont été observés dans toutes les variétés. Cependant, les deux pics $n^{\circ} 8$ (butyrate d'éthyle) et $\mathrm{n}^{\circ} 10$ (2-méthyle butyrate d'éthyle) observés à 4,8 min et 5,3 min, importants chez les fruits de Perolera, de Queen et des hybrides Cayenne $\times$ Perolera, ont été faibles chez les fruits de Cayenne et de Rondon et très faibles pour l'hybride MD2 (tableau IV).

Pour la famille F2, caractérisée par des temps de rétention de $(7,4$ à 11,8$) \mathrm{min}$, les \% SI ont été moyennement homogènes (tableau III). Cette famille comporte peu de pics, mais deux sont très intenses : le pic $\mathrm{n}^{\circ} 1$ correspondant à l'hexanoate de méthyle et le pic $n^{\circ} 3$, à l'hexanoate d'éthyle, observés respectivement à $(7,8$ et à 8,6$)$ min (figure 1). Pour les fruits de Queen et de Perolera, ces deux pics ont été remarquablement plus grands que pour ceux des autres variétés (tableau IV). Chez les fruits du clone Qu2 des ananas Queen en particulier, ils représentent près de $60 \%$ SI et chez ceux du clone Qu3, près de $50 \%$ SI, alors que chez les fruits du clone Qu1 ils ne
Tableau II.

Nature des principaux composés aromatiques de chacune des quatre familles résultant du découpage des chromatogrammes issus de l'utilisation d'une analyse SPME, en fonction du temps de rétention de ces composés volatils $[F 1$, temps de rétention de $(0$ à $7,4) \min ; F 2$, de $(7,4$ à 11,8$) \min ; F 3$, de $(11,8$ à 15,1$) \min ; F 4$, de $(15,1$ à 25$) \mathrm{min}]$.

$\begin{array}{lc}\text { Famille } & \text { Composés volatils } \\ \text { F1 } & \begin{array}{c}\text { Esters d'acétate et de butyrate } \\ \text { F2 }\end{array} \\ \text { F3 } & \begin{array}{c}\text { Esters de pentanoate, d'hexanoate et heptanoate } \\ + \text { quelques terpènes }\end{array} \\ \text { F4 } & \begin{array}{c}\text { Esters d'octanoate et octénoate + sesquiterpènes } \\ \text { Esters nonanoate et décanoate } \\ \end{array} \\ \text { Thioesters } \\ \text { Sesquiterpènes }\end{array}$

représentent que $25 \%$ SI (tableau III). Chez les fruits hybrides, les \% SI ont moins varié, de $(26,5$ à 39,9) \% SI, alors que chez les fruits de Cayenne, ces valeurs se sont distribuées entre $(21,1$ et 42,3$) \%$ SI. Le MD2 présente un résultat un peu moindre de 35,4\% SI, alors que les fruits de Rondon ont présenté des résultats plus faibles, de $(10,1$ à 26,6) \% SI, à l'exception des fruits du clone Ro5, avec 39 \% SI. Ce dernier s'est distingué cependant par une faible intensité de ses arômes, traduite par l'observation de la surface d'intégration totale la plus faible.

Pour la famille F3, caractérisée par des temps de rétention de $(11,8$ à 15,1$) \mathrm{min}$, les $\%$ SI ont été beaucoup plus variables que pour ceux de la famille F2, cela quelle que soit la variété considérée et particulièrement pour les fruits de Cayenne lisse qui ont présenté de $(17,9$ à 40,8) \% SI. Pour les fruits de Queen, les valeurs ont été plus faibles, de l'ordre de $20 \%$ SI (tableau III). Pour les fruits de Rondon et de Perolera, il a été noté la quasi absence du pic $n^{\circ} 2$ au temps de rétention de $12,8 \mathrm{~min}$, correspondant à un sesquiterpène $\alpha$-cubébène (tableau IV). Les deux pics majeurs de cette famille F3, le pic $\mathrm{n}^{\circ} 1$ correspondant à l'octanoate de méthyle et le pic n 3 , octanoate d'éthyle, avec, respectivement, des temps de rétention de $(12,2$ et 13,2$)$ min (figure 1), ont été beaucoup plus importants chez les fruits de Perolera, Rondon et Cayenne et inversement, faibles, chez les hybrides Flhoran 41 et MD2. 


\begin{tabular}{|c|c|c|c|c|c|}
\hline & $\begin{array}{l}\text { Tableau III. } \\
\text { Variations inter- et intravariétales prés } \\
\text { de composés volatils, par rappor } \\
\text { d'intégration (SI) pour l'analyse des fru } \\
\text { de différentes variétés d'Ananas com } \\
\text { de (0 à } 7,4) \text { min ; F2, de }(7,4 \text { à } 11,8) \mathrm{m} \\
\mathrm{F} 4 \text {, de }(15,1 \text { à } 25) \text { min]. } \\
\text { Variété-clone Surface d'intégration totale } \\
\text { (unités d'intégration) }\end{array}$ & & 12 & & $\mathrm{~F} 4$ \\
\hline $\mathrm{Ca} 1$ & 672592 & 1,9 & 27,5 & 16,9 & 53,1 \\
\hline $\mathrm{Ca} 2$ & 1749202 & 4,8 & 42,3 & 34,4 & 18,5 \\
\hline $\mathrm{Ca} 3$ & 2109640 & 5,6 & 32,4 & 39,5 & 22,6 \\
\hline Ca4 & 2944282 & 6,2 & 28,3 & 40,8 & 24,7 \\
\hline Ca5 & 699113 & 1,3 & 21,1 & 17,9 & 58,4 \\
\hline \multicolumn{6}{|l|}{ Perolera } \\
\hline $\mathrm{Pe} 1$ & 561528 & 7,1 & 44,9 & 41,2 & 6,8 \\
\hline $\mathrm{Pe} 2$ & 1747482 & 4,7 & 53,2 & 33,5 & 8,6 \\
\hline $\mathrm{Pe} 3$ & 1668261 & 25,4 & 55,1 & 15,3 & 4,1 \\
\hline $\mathrm{Pe} 4$ & 3777811 & 5,1 & 24,5 & 60,5 & 9,9 \\
\hline \multicolumn{6}{|l|}{ Queen } \\
\hline Qu1 & 5554529 & 10,3 & 25,3 & 35,2 & 14,7 \\
\hline Qu2 & 3012870 & 3,7 & 58,0 & 23,5 & 14,7 \\
\hline Qu3 & 2817635 & 5,1 & 48,9 & 16,4 & 18,0 \\
\hline \multicolumn{6}{|l|}{ Rondon } \\
\hline Ro1 & 811586 & 7,7 & 26,6 & 43,9 & 21,9 \\
\hline Ro2 & 3187101 & 9,0 & 10,4 & 36,3 & 44,3 \\
\hline Ro3 & 2475838 & 5,5 & 10,1 & 49,3 & 35,2 \\
\hline Ro4 & 941183 & 7,3 & 17,5 & 40,6 & 34,6 \\
\hline Ro5 & 247470 & 3,3 & 39,3 & 35,9 & 21,5 \\
\hline \multicolumn{6}{|c|}{ Hybride Cayenne $\times$ Perolera } \\
\hline Hy1 & 1989670 & 10,2 & 39,9 & 26,7 & 23,2 \\
\hline Hy2 & 815098 & 7,2 & 34,9 & 42,1 & 15,8 \\
\hline Нy3 & 820019 & 4,9 & 34,3 & 34,7 & 26,1 \\
\hline $\mathrm{Hy} 4$ & 2342343 & 0,7 & 26,5 & 42,8 & 30,1 \\
\hline Flhoran 41 & 1425976 & 12,3 & 32,1 & 25,8 & 29,8 \\
\hline \multicolumn{6}{|c|}{ Hybride autre origine } \\
\hline MD2 & 916710 & 11,5 & 40,3 & 23,8 & 24,4 \\
\hline
\end{tabular}

Pour la famille F4, caractérisée par des temps de rétention de (15 à 25) min, les \% SI ont globalement varié de (15 à 35) \% SI. Ils ont été faibles et homogènes pour les fruits de Queen (15\% SI), et faibles et hétérogènes pour ceux de Perolera: (de 4 à 10) \% SI (tableau III). Les fruits de Perolera et Rondon ont révélé moins de pics pour les composés de ce type que ceux des autres variétés et, pour les Rondons, les trois pics, $\mathrm{n}^{\circ} 1$ à 16,8 min (décanoate de méthyle), $\mathrm{n}^{\circ} 2$ à 17,6 min (caprate d'éthyle) et $\mathrm{n}^{\circ} 3$ à 19,0 min (sesquiterpène : $\alpha$-murolène) sont apparus plus intenses que pour les fruits des autres variétés étudiées. Les fruits des hybrides ont présenté des valeurs variant de $(23,2$ à 30,1) \% SI, sauf pour les fruits de Hy2, présentant des valeurs beaucoup plus faibles : $15 \%$ SI. De nombreux pics aromatiques de faible intensité composent cette famille (figure 1).

Globalement, les profils aromatiques des différentes variétés sont proches les uns des autres en terme de nombre de pics et d'intensité, à quelques exceptions près pour certains clones.

Les fruits de Queen se sont démarqués de ceux des autres variétés par une forte production de composés volatils appartenant à la famille F2 (58 \% SI) et une très forte homogénéité intravariétale (figure 1). Cela confirmerait des analyses sensorielles qui ont montré que les consommateurs trouvaient les fruits de Queen très aromatiques, en plus d'être très sucrés, mais qui n'ont pu permettre de différentier les différents clones entre eux [17].

Les fruits de Perolera sont également très homogènes d'un clone à l'autre, mais cela dans une moindre mesure que les fruits de Queen.

Les fruits des différents clones de Cayenne et de Rondon se sont révélés un peu moins homogènes entre eux que ceux des ananas Queen et Perolera.

Les fruits hybrides ont révélé une certaine hétérogénéité entre eux ce qui est cohérent avec leur origine. Ils ont présenté trois grands pics dans la famille F2 à peu près identiques en intensité et qui les démarquent nettement des fruits de leurs parents Cayenne et Perolera. Les différents hybrides ont montré des flaveurs, saveurs et des teneurs en sucres et acides très différentes les uns des autres [17]. 
Les fruits de Rondon ont présenté un grand nombre de pics dans la famille F1 et les grands pics observés pour la famille F2 ont été faibles par rapport aux fruits des autres variétés. Les composés aromatiques plus "lourds " (familles F3 et F4) ont été les plus représentés. Dans la famille F4, peu de pics ont été observés pour les fruits de cette variété, mais ils ont été de plus forte intensité que ceux notés chez les autres variétés (tableau IV).

\subsection{Effet de la maturation sur les profils aromatiques de Flhoran 41}

Pour les fruits de l'hybride Flhoran 41, l'émission aromatique très faible à $147 \mathrm{j}$ après le traitement d'induction florale (TIF), stade de récolte pour l'exportation en frais par bateau, a augmenté très fortement de (151 à 155) j après TIF. À 155 j après TIF, elle a été maximale, puis, à 158 j, elle a diminué, les fruits étant alors sur-mûrs (tableau V). Il n'y a pas eu de différence qualitative majeure entre les fruits ayant atteint les stades de maturité de 151 j et ceux de $158 \mathrm{j}$, mais plus les fruits ont mûri, plus les composés lourds et moins volatils (famille F4) se sont développés.

\subsection{Effet de la conservation à $24^{\circ} \mathrm{C}$ sur les profils aromatiques de Queen}

Sur des fruits de Queen verts ou en tout début de maturation, une forte production de composés volatils a eu lieu après $8 \mathrm{j}$ de conservation, six à sept fois plus intense que celle observée à la récolte (tableau VI). Elle est toutefois restée inférieure à la production obtenue à pleine maturation.

\subsection{Effet de la conservation type " export bateau" sur les profils aromatiques du Flhoran 41}

Pour des fruits de l'hybride Flhoran 41, la production de composés aromatiques a été multipliée par 30 après 13 j en conteneurs à $18^{\circ} \mathrm{C}$, puis cette dernière valeur a été multipliée par 2,5 après $7 \mathrm{j}$ supplémentaires à $13{ }^{\circ} \mathrm{C}$. Elle est ensuite restée stable

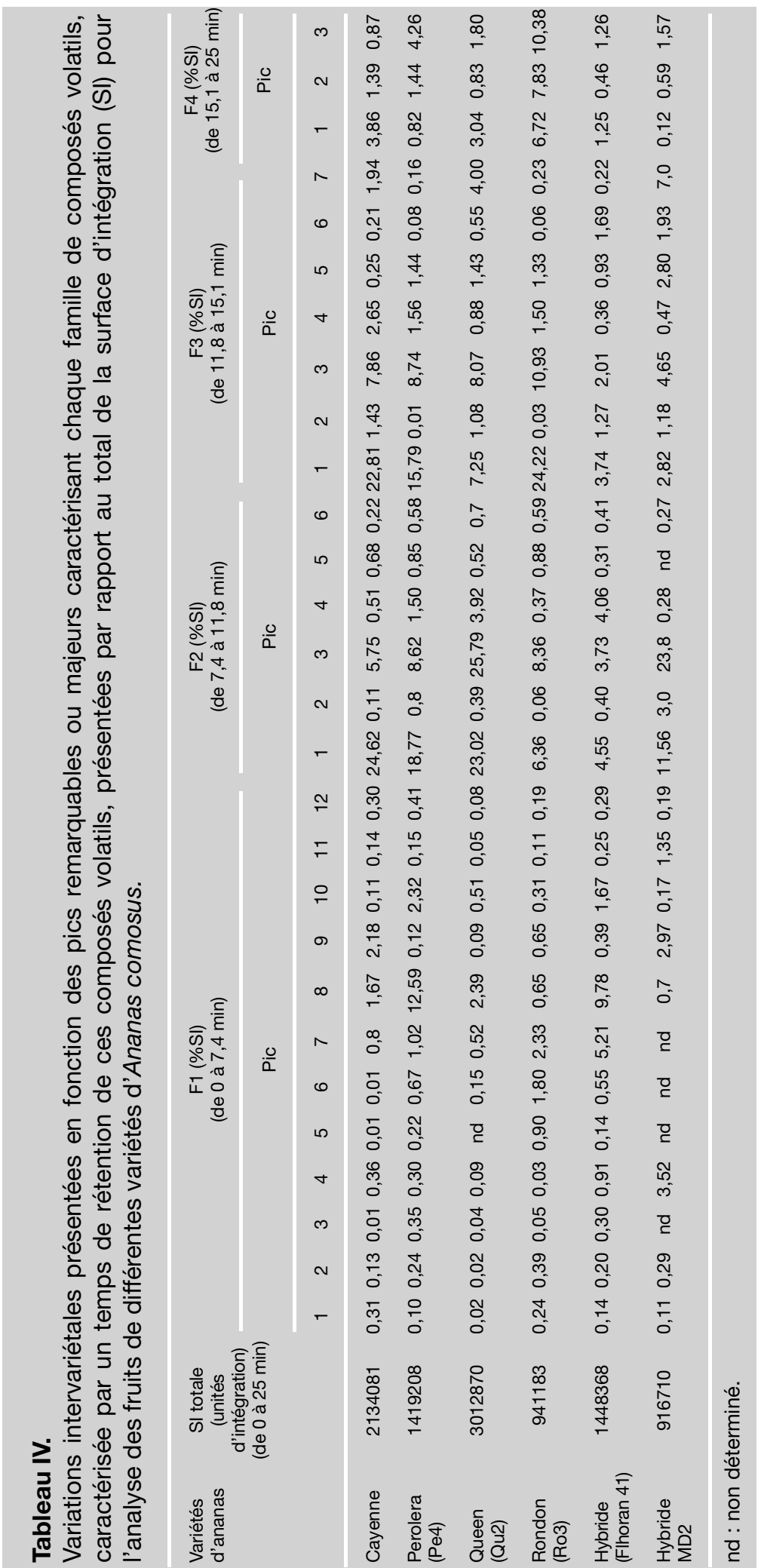

Fruits, vol. 61 (3) 


\section{Tableau V.}

Émission aromatique des fruits de l'hybride Flhoran 41 (Ananas comosus) en maturation naturelle, en fonction du stade récolte évalué en nombre de jours après le traitement d'induction florale (TIF). Mise en relation du taux de la surface d'intégration totale des familles de composés volatils du chromatogramme aromatique avec les paramètres extrait sec soluble et acidité libre $[F 1$, temps de rétention de $(0$ à 7,4$) \min ; F 2$, de $(7,4$ à 11,8$) \min$; $\mathrm{F} 3$, de $(11,8$ à 15,1$) \mathrm{min} ; \mathrm{F} 4$, de $(15,1$ à 25$) \mathrm{min}]$.

\begin{tabular}{lccccccc}
$\begin{array}{l}\text { Stade } \\
\text { récolte } \\
\text { (j après TIF) }\end{array}$ & $\begin{array}{c}\text { Extrait sec soluble } \\
\left({ }^{\circ} \text { Brix }\right)\end{array}$ & $\begin{array}{c}\text { Acidité libre } \\
\left(\mathrm{mEq} \cdot 100 \mathrm{~mL}^{-1}\right)\end{array}$ & $\begin{array}{c}\text { Surface d'Intégration totale } \\
\text { (unités d'intégration) }\end{array}$ & Famille 1 & Famille 2 & Famille 3 & Famille 4 \\
\hline 147 & 15,5 & 11,5 & 85869 & 28,1 & 36,0 & 23,0 & 13,0 \\
151 & 16,1 & 13,1 & 344008 & 28,2 & 33,3 & 15,8 & 21,1 \\
155 & 16,6 & 12,7 & 1676375 & 27,5 & 20,7 & 16,2 & 35,5 \\
158 & 17,6 & 12,0 & 518092 & 28,6 & 25,9 & 11,5 & 34,1
\end{tabular}

\section{Tableau VI.}

Effet de la conservation à $24{ }^{\circ} \mathrm{C}$ sur les profils aromatiques de fruits de Queen (Ananas comosus).

a) Caractéristiques des lots analysés.

\begin{tabular}{lccccc} 
Lot analysé & Maturité & $\begin{array}{c}\text { Conservation } \\
\text { (j) }\end{array}$ & $\begin{array}{c}\text { Température } \\
\left({ }^{\circ} \mathrm{C}\right)\end{array}$ & $\begin{array}{c}\text { Acidité libre } \\
\left(\mathrm{g} \cdot 100 \mathrm{~mL}^{-1}\right)\end{array}$ & $\begin{array}{c}\text { Extrait sec } \\
\left({ }^{\circ} \mathrm{Brix}\right)\end{array}$ \\
\hline Lot 1 & Vert & Témoin & - & 6,32 & 10,4 \\
Lot 2 & Vert & +8 & 24 & 10,30 & 12,2
\end{tabular}

b) Émission aromatique (unités d'intégration) pour chacune des familles F1 à F4 de composés volatils du chromatogramme obtenu après analyse des fruits par SPME.

\begin{tabular}{lccccc} 
Lot analysé & $\begin{array}{c}\text { Surface d'intégration totale } \\
\text { (0 à } 25 \mathrm{~min})\end{array}$ & $\begin{array}{c}\mathrm{F} 1 \\
(0 \text { à } 7,4 \mathrm{~min})\end{array}$ & $\begin{array}{c}\mathrm{F} 2 \\
(7,4 \text { à } 11,8 \mathrm{~min})\end{array}$ & $\begin{array}{c}\mathrm{F} 3 \\
(11,8 \text { à 15,1 min) }\end{array}$ & $\begin{array}{c}\mathrm{F} 4 \\
(15,1 \text { à 25 min) }\end{array}$ \\
\hline Lot 1 & 38677 & 9418 & 11173 & 6722 & 17966 \\
Lot 2 & 260820 & 44388 & 70472 & 75090 & 66586
\end{tabular}

(tableau VII). Comme dans le cas de fruits laissés en maturation naturelle, le développement de composés " lourds " des familles $\mathrm{F} 3$ et $\mathrm{F} 4$ a prédominé après 13 j de conservation. Ce fort développement des composés volatils au cours de la conservation des fruits ne compense pas cependant l'absence de saveur liée à une faible maturité avec une faible teneur en sucres.

\subsection{Effet terroir sur le Flhoran 41}

La production de l'hybride Flhoran 41 sur un site en altitude induit une réduction de la vitesse de maturation des fruits. La peau des fruits produit alors une quantité d'anthocyanes plus importante donnant ainsi des fruits de couleur rouge au lieu de fruits plus orangés observés en basse altitude [18]. Pour l'analyse aromatique, les fruits sont récoltés à des dates différentes mais à un stade de maturité équivalent mesuré par l'extrait sec des solides solubles et l'acidité libre. Le terroir n'a pas induit de différences qualitatives significatives, les mêmes composés volatils ont été observés bien que, pour les fruits produits en altitude, l'importance relative des familles F1 et F2 ait été un peu plus faible (tableau VIII). 


\section{Tableau VII.}

Effet de la conservation type « export bateau » sur les profils aromatiques des fruits de l'hybride Flhoran 41 (Ananas comosus).

a) Caractéristiques des lots analysés.

$\begin{array}{lccccc}\text { Lot analysé } & \text { Maturité } & \begin{array}{c}\text { Traitement de conservation } \\ \text { Lot } 1\end{array} & \begin{array}{c}\text { Température } \\ \left({ }^{\circ} \mathrm{C}\right)\end{array} & \begin{array}{c}\text { Acidité libre } \\ \left(\mathbf{g} \cdot 100 \mathrm{~mL}^{-1}\right)\end{array} & \begin{array}{c}\text { Extrait sec } \\ \left({ }^{\circ} \mathrm{Brix}\right)\end{array} \\ \text { Lot 2 } & \text { Mûr } & \text { Témoin } & - & 11,5 & 15,5 \\ \text { Lot 3 } & \text { Mûr } & +13 & 18 & 19,1 & 15,5 \\ \text { Lot } 4 & \text { Mûr } & +7 & 13 & 17,2 & 15,4 \\ & \text { Mûr } & +5 & 24 & 13,8 & 14,3\end{array}$

b) Émission aromatique pour chacune des familles F1 à F4 de composés volatils du chromatogramme obtenu après analyse des fruits par SPME, exprimée en \% de la surface d'intégration totale (SI).

\begin{tabular}{|c|c|c|c|c|c|}
\hline \multirow[t]{2}{*}{ Lot analysé } & \multirow{2}{*}{$\begin{array}{l}\text { Surface d'intégration totale } \\
\text { (0 à } 25 \text { min) } \\
\text { (unités d'Intégration) }\end{array}$} & $\begin{array}{c}\mathrm{F} 1 \\
(0 \text { à } 7,4 \mathrm{~min})\end{array}$ & $\begin{array}{c}\mathrm{F} 2 \\
(7,4 \text { à } 11,8 \mathrm{~min})\end{array}$ & $\begin{array}{c}\mathrm{F} 3 \\
(11,8 \text { à } 15,1 \mathrm{~min})\end{array}$ & $\begin{array}{c}\mathrm{F} 4 \\
(15,1 \text { à } 25 \mathrm{~min})\end{array}$ \\
\hline & & \multicolumn{4}{|c|}{ (\% SI) } \\
\hline Lot 1 & 85869 & 28,1 & 36,0 & 23,0 & 13,0 \\
\hline Lot 2 & 2705789 & 21,4 & 27,8 & 28,1 & 21,6 \\
\hline Lot 3 & 7070676 & 16,5 & 14,0 & 41,5 & 21,6 \\
\hline Lot 4 & 8917958 & 16,4 & 10,8 & 34,5 & 34,1 \\
\hline
\end{tabular}

Tableau VIII.

Effet « terroir » (altitude et sols différents) sur la qualité des fruits de l'hybride Flhoran 41 (Ananas comosus) (Martinique).

a) Caractéristiques des fruits analysés.

\begin{tabular}{lcccccc}
\hline Localisation & $\begin{array}{c}\text { Altitude } \\
(\mathrm{m})\end{array}$ & Jour de récolte & Jour d'analyse & Maturité & $\begin{array}{c}\text { Acidité libre } \\
\left(\mathrm{g} \cdot 100 \mathrm{~mL}^{-1}\right)\end{array}$ & $\begin{array}{c}\text { Extrait sec } \\
\left({ }^{\circ} \text { Brix }\right)\end{array}$ \\
\hline $\begin{array}{l}\text { Rivière-Lézarde } \\
\text { Basse-Pointe }\end{array}$ & 50 & 18 juin 2003 & 20 juin 2003 & Mûr & 11,07 & 17,5 \\
\hline
\end{tabular}

b) Émission aromatique pour chacune des familles F1 à F4 de composés volatils du chromatogramme obtenu après analyse des fruits par SPME, exprimée en \% de la surface d'intégration totale (SI).

\begin{tabular}{|c|c|c|c|c|c|}
\hline \multirow[t]{2}{*}{$\begin{array}{l}\text { Altitude } \\
\text { (m) }\end{array}$} & \multirow{2}{*}{$\begin{array}{l}\text { Surface d'intégration totale } \\
\text { ( } 0 \text { à } 25 \mathrm{~min} \text { ) } \\
\text { (unités d'intégration) }\end{array}$} & $\begin{array}{c}\mathrm{F} 1 \\
(0 \text { à } 7,4 \mathrm{~min})\end{array}$ & $\begin{array}{c}\mathrm{F} 2 \\
(7,4 \text { à } 11,8 \mathrm{~min})\end{array}$ & $\begin{array}{c}\mathrm{F} 3 \\
(11,8 \text { à } 15,1 \mathrm{~min})\end{array}$ & $\begin{array}{c}F 4 \\
(15,1 \text { à } 25 \mathrm{~min})\end{array}$ \\
\hline & & \multicolumn{4}{|c|}{ (\% SI) } \\
\hline 50 & 1397081 & 23,8 & 10,1 & 33,0 & 33,1 \\
\hline 135 & 2464762 & 13,3 & 28,8 & 33,9 & 24,0 \\
\hline
\end{tabular}

\section{Conclusion}

Différencier les profils aromatiques par SPME de différentes variétés d'ananas semble possible malgré leur grande similitude.
Une identification plus poussée, par GC-MS, des composés volatils d'ananas de différentes origines pourrait permettre de mieux préciser leur caractérisation. Elle conduirait aussi à mieux expliquer l'évolution des 
arômes au cours de la conservation et du transport des fruits. L'état de maturité est particulièrement important pour effectuer des comparaisons intra ou intervariétales. Une analyse comparative entre les résultats obtenus par extraction en SPME et une extraction classique [liquide : liquide] est en cours.

\section{Références}

[1] Horry J.P., Une collection mondiale d'ananas, Cah. PRAM 1 (2003) 26-27.

[2] Anon., Plant variety rights act: 'FLHORAN41', Cirad-Flhor, Community Plant Var. Off., 2004, 12638.

[3] Soler A., Amélioration des systèmes de production en culture d'ananas: analyses aromatiques de variétés d'ananas, Cirad-Flhor, Rapp. tech. exéc. (annexe 1), Fort-deFrance, Martinique, France, 2003.

[4] Berger R.G., Drawert F., Kollmannsberger H., Nitz S., Schraufstetter B., Novel volatiles in pineapple fruit and their sensory properties, J. Agric. Food Chem. 33 (1985) 232-235.

[5] Teai T., Claude-Lafontaine A., Schippa C., Cozzolino F., Volatile compounds in fresh pulp of pineapple (Ananas comosus [L.] Merr.) from French Polynesia, J. Essent. Oil Res. 13(5) (2001) 314-318.

[6] Brat P., Thi Hoang L.N., Soler A., Reynes M., Brillouet J.M., Physicochemical characterization of a new pineapple hybrid (Flhoran 41 cv.), J. Agric. Food Chem. 52 (2004) 61706177.

[7] Takeoka G.R., Buttery R.G., Teranishi R., Flath R.A., Günter M., Identification of additional pineapple volatiles, J. Agric. Food Chem. 39 (1991) 1848-1851.

[8] Rodin J.O., Himel C.M., Silverstein R.M., Leeper R.W., Gortner W.A., Volatile flavor and aroma components of pineapple. 1. Isolation and tentative identification of 2,5-dimethyl-4hydroxy-3[2H]-furanone, J. Food Sci. 30 (1965) 280-285.

[9] Anon., Introduction générale à l'examen de la distinction, de l'homogénéité et de la stabilité et à l'harmonisation des descriptions des obtentions végétales, TG/1/3, UPOV, Genève, 2002, 27 p.

[10] Elss S., Preston C., Hertzig C., Richling E., Schreier P., Über das Aromaprofil der Ananas (Ananas comosus [L.] Merr.). Eine NachernteStudie, Flüssiges Obst. 70 (12) (2003) 734737.

[11] Cardeal Z. de L., Guimaraes E.M., Parreira F.V., Analysis of volatile compounds in some typical Brazilian fruits and juices by SPMEGC method, Food Addit. Contam. 22 (6) (2005) 508-513.

[12] Jirovetz L., Buchbauer G., Geissler M., Ngassoum M.B., Parmentier M., Pulp aroma compounds of untreated, boiled and roasted African pear [Dacryodes edulis (G. Don) H.J. Lam] fruits from Cameroon by HS-SPME analysis coupled with GC/FID and GC/MS, Eur. Food Res. Technol. 218 (1) (2004) 40-43.

[13] Lalel H.J.D., Singh Z., Tan S.C., Distribution of aroma volatile compounds in different parts of mango fruit, J. Hort. Sci. Biotechnol. 78 (2) (2003) 131-138.

[14] Bravdo B., Shoseyov O., Aroma studies of fruits and wine in Israel, Acta Hortic. (ISHS) (526) (2000) 399-406.

[15] Augusto F., Valente A.L., dos Santos Tada E., Rivellino S.R., Screening of Brazilian fruit aromas using solid-phase microextraction-gas chromatography-mass spectrometry, J. Chromatogr. A 873 (1) (2000) 117-127.

[16] Umano K., Hagi Y., Nakahara K., Shoji A., Shibamoto T., Volatiles constituents of green and ripened pineapple (Ananas comosus [L.] Merr.), J. Agric. Food Chem. 40 (1992) 599603.

[17] Bertrand M., Le Flhoran 41 et les autres nouvelles variétés d'ananas au Cirad: essais de conservation au froid, de transformation et tests sensoriels de dégustation, Univ. Paris XII-Créteil, DESS Gestion des systèmes agro-sylvo-pastoraux en zones tropicales, Mém., Créteil, France, 2004.

[18] Soler A., Fournier P., Determination of anthocyanin biosynthesis in the Cirad pineapple hybrid Flhoran 41, in: Proc. V Int. Pineapple Symp., ISHS, 2005 (in press). 


\section{Caracterización del perfil aromático de diferentes variedades de piñas por SPME en espacio de cabeza / GC-FID.}

Resumen - Introducción. Entre las variedades de piñas (Ananas comosus [L.] Merr.) la variabilidad de las características sensoriales es mínima entre clones pero más amplia entre variedades. Diez híbridos Cayena $\times$ Perolera han sido seleccionados por el Cirad a fines comerciales. Muestran unos perfiles aromáticos distintos de los padres. La piña debe ser cosechada relativamente verde para llegar en buenas condiciones a los mercados consumidores. Como el flavor de la piña puede conllevar al consulidor a comprarla, hemos estudiado en diferentes variedades e híbridos, el impacto de la maduración de la fruta y de las condiciones de conservación sobre la emisión de compuestos volátiles. Material y métodos. Los niveles de compuestos volátiles de las diferentes variedades pudieron ser medidos, tras extracción por SPME (fibra PDMS $100 \mu \mathrm{m}$ ), por CPG/FID sobre columna Stabilwax. Los análisis se refieren a frutas de cuatro o cinco clones de cada variedad Cayena, Perolera, Queen y Rondon, así como cinco híbridos Cirad y otro híbrido MD2. El impacto del nivel de maduración, medido por los sólidos solubles y la acidez, y de las condiciones de conservación sobre la emisión de compuestos volátiles ha sido evaluado. Resultados y discusión. Los perfiles aromáticos obtenidos para diferentes clones de una misma variedad son muy similares mientras las diferencias entre variedades fueron reproductibles. Durante la maduración, una emisión de compuestos volátiles se produjo antes la maduración completa. La conservación al frío entre $13{ }^{\circ} \mathrm{C}$ y $24{ }^{\circ} \mathrm{C}$ conlleva a una fuerte emisión de compuestos volátiles. Conclusión. Diferenciar perfiles aromáticos por SPME/GC-FID parece posible a pesar de ser muy parecidos. Una identificación más profunda por GC-MS de los compuestos volátiles de pina de diferentes orígenes permitiría una mejor caracterización.

Martinica / Ananas comosus / ensayos de variedades / cromatografía de gases / compuesto volátil / maduración / almacenamiento en frío 